\title{
Complications and Pitfalls in Maxillofacial Trauma
}

\author{
${ }^{1}$ Vidya Rattan, ${ }^{2}$ Sachin Rai, ${ }^{3}$ Shikha Yadav
}

\begin{abstract}
Complications and pitfalls are unavoidable sequel of any surgical procedure. Although advances have been made in surgical techniques and fixation devices, these are far from ideal. The aim of trauma surgeon is to restore the patient to pre-injury form and function, but it may not be possible in each and every case. Treatment failure and poor outcome in a case of severe maxillofacial trauma is not uncommon. This is mainly dependent upon the extent of injury, delay in management, improper diagnosis, wrong treatment plan, poor execution of a treatment plan, wrong choice of fixation devices, inexperience of the surgeon and inability to seek help from other specialists, etc. The objective of this article is to discuss and analyze common complications and pitfalls along with the various methods and modalities to prevent and manage it.
\end{abstract}

Keywords: Maxillofacial trauma, Complications, Malunion, Nonunion.

How to cite this article: Rattan V, Rai S, Yadav S. Complications and Pitfalls in Maxillofacial Trauma. J Postgrad Med Edu Res 2014;48(2):91-97.

Source of support: Nil

Conflict of interest: None

\section{INTRODUCTION}

Complications and pitfalls are part and parcel of any surgical procedure but the goal of a surgeon should be to minimize them. Although advances have been made in surgical techniques and fixation devices, these are far from ideal. The aim of trauma surgeon is to restore the patient to the preinjury form and function, but it may not be possible in each and every case. Treatment failure and poor outcome in a severe case of maxillofacial trauma is not uncommon. This is mainly dependent upon the extent of injury, delay in management, improper diagnosis, wrong treatment plan, poor execution of a treatment plan, wrong choice of fixation devices, inexperience of the surgeon and inability to seek help from other specialists, etc. The objective of this article is to discuss and analyze common complications and pitfalls,

\footnotetext{
${ }^{1}$ Additional Professor, ${ }^{2}$ Assistant Professor, ${ }^{3}$ Senior Resident

${ }^{1-3}$ Unit of Oral and Maxillofacial Surgery, Oral Health Sciences Centre, Postgraduate Institute of Medical Education and Research, Chandigarh, India
}

Corresponding Author: Sachin Rai, Assistant Professor Unit of Oral and Maxillofacial Surgery, Oral Health Sciences Centre Postgraduate Institute of Medical Education and Research Chandigarh, India, Phone: 0172-2756261, e-mail: drraisachin@ gmail.com along with the various methods and modalities to prevent and manage it. The problems associated with maxillofacial trauma management will be discussed under following headings (Table 1 ).

Table 1: Complications associated with management of maxillofacial trauma

1 Complications in fracture healing

2 Complications related to fixation devices

3 Postoperative facial deformity

4 Ophthalmic complications

5 Infections

6 Hard and soft tissue loss

7 Growth and TMJ problems

8 Nerve injury

\section{COMPLICATIONS IN FRACTURE HEALING}

Complications associated with healing of fractures can be divided into delayed union, nonunion and malunion. Delayed union and nonunion are the fractures where fracture fails to heal in 2 to 3 months. Delayed unions go on to heal without additional surgical therapy, whereas nonunion is a fracture with arrested healing that requires further surgical therapy to achieve union. Malunion is a clinical term which indicates that a fracture has healed in less than an optimal position. Malunion may result in a bone being shorter than normal, twisted or rotated in a wrong position. This results from the inability to reduce the bony fragments in proper position or failure of fixation devices to hold the reduced fragments in proper position. The prevention of delayed union and nonunion lies in proper diagnosis and adequate use of fixation devices, depending on the case. The technique and devices need to be modified in poor bone stock situations and in cases where poor healing is anticipated. Figures $1 \mathrm{~A}$ to $\mathrm{C}$ depict a case where inadequate fixation in case of comminuted fracture led to nonunion and osteomyelitis. The necrotic bone was removed, and adequate fixation was applied which led to healing of fracture.

Malunion of facial bones leads to malocclusion and disfigurement. Interception of malocclusion needs to be done as early as possible and reduction within the earliest possible time frame so that the optimal function is restored and skeletal and soft tissue deformity is minimized. ${ }^{1}$ Preinjury records like radiographs and photographs provide 
a good baseline data for the treatment planning. Facial deformity and malocclusion can be managed with conventional orthognathic surgical and orthodontic procedures. ${ }^{2}$ It is a team work between maxillofacial surgeon, orthodontist and prosthodontist. Correction of deformities caused by malunion will be further discussed in management of facial deformity.

\section{COMPLICATIONS RELATED TO FIXATION DEVICES}

Complications related to fixation devices are multifactorial. It can be screw loosening, breakage, metal sensitivity, thermal sensitivity, tooth injury, nerve injury, malocclusion or growth restrictions. Metal devices used for osteosynthesis though biocompatible, are foreign bodies and do not behave like natural tissues and can cause altered sensory reaction to thermal and various other stimuli. The trauma surgeon should be familiar with technique and limitations of the devices being used for particular fracture treatment.

Common infringements of rigid fixation principles include, e.g. a plate that is too small, one plate instead of two, placement of a screw into the line of fracture, too few screws per side of fracture and inadequate plate bending. Overheating that could potentially lead to bone necrosis and hardware failure, therefore adequate irrigation while drilling is essential.

Zachariades et al compared the complication rates between rigid fixation with plates vs interosseous wires in mandibular and midfacial fractures over a 6 -year period. ${ }^{3}$ The complica- tions examined included infection (early and late), delayed union, malocclusion, hypoesthesia, wound dehiscence and disfigurement. The overall complication rate with rigid fixation was $12.8 \%$, compared with a rate of $17.8 \%$ with interosseous wiring. Bioresorbable osteosynthesis system have been developed to overcome some of the problems associated with metallic devices and have been used with good success in certain conditions. These provide normal growth and development of jaw bone and eliminate the indication of second surgery for removal. In future if any imaging is required it does not cause interference. Lack of rigidity compared to metallic plates, makes them less ideal to be used in all maxillofacial trauma situations. ${ }^{4}$

\section{POST-TRAUMATIC FACIAL DEFORMITY}

Trauma to face if not treated adequately can cause obvious disfigurement and even minor changes on face are quite visible and noticeable. Therefore, perfect reduction of fractures is of paramount importance in case of facial bone fractures. Extent of facial deformity is site specific and depends on extent of malpositioning of fractured bone. Deformity can manifest as asymmetry, loss of facial height, facial width problems, loss of projection and loss of occlusion. Facial deformity of soft or hard tissue can be a result of diagnostic errors, poor surgical technique, inadequate reductions and fixation or no treatment at all. Complications associated with nasoethmoidal injuries can be saddle nose, anosmia, telecanthus, unsightly scars, enophthalmos, diplopia, nasolacrimal obstruction, CSF leak, and sinusitis, etc. Corrrection

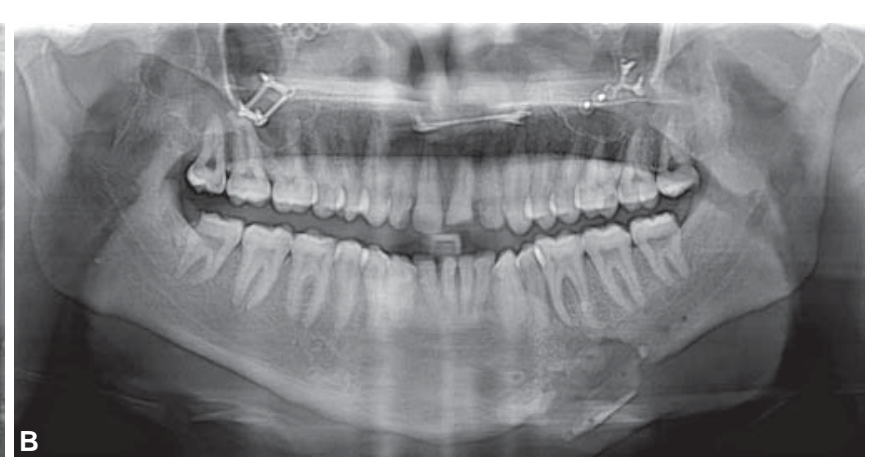

B
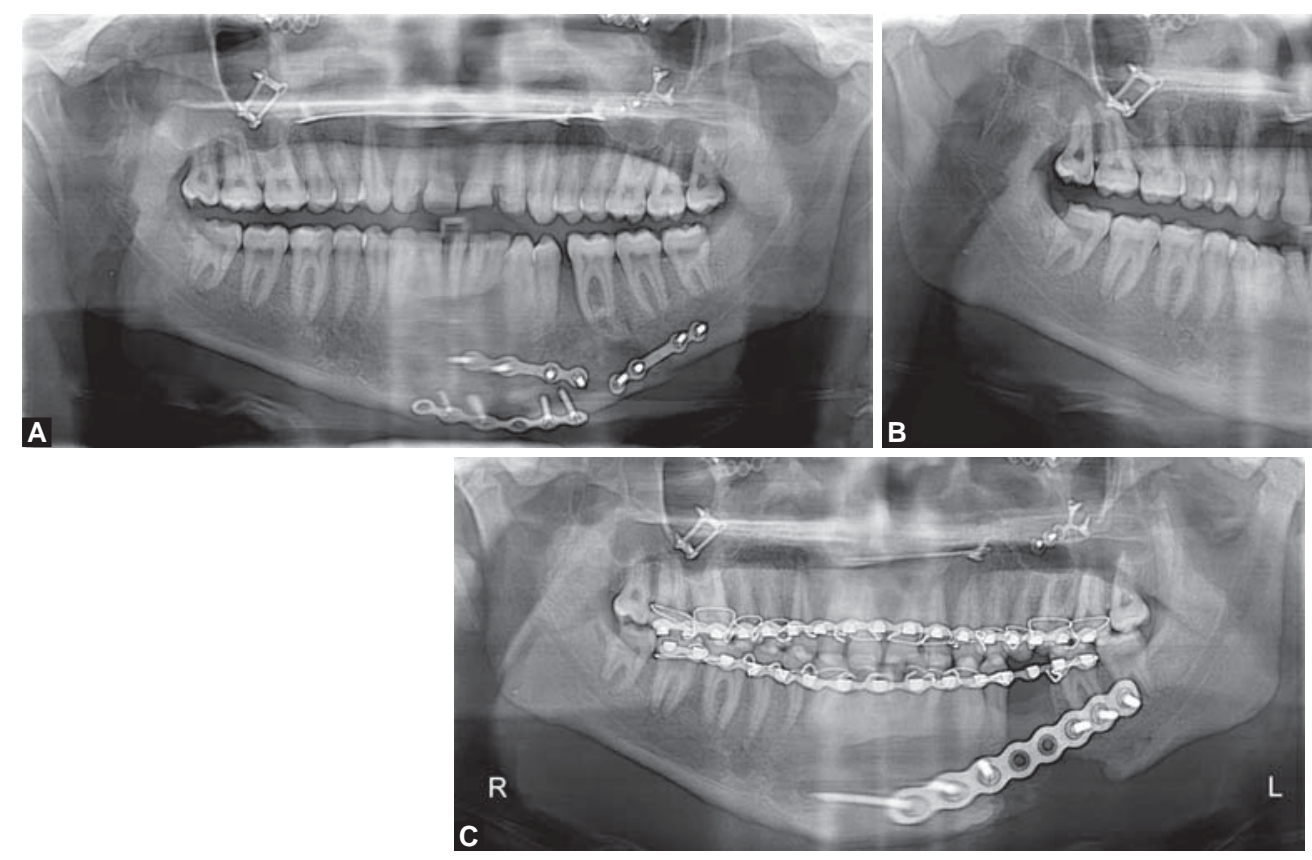

Figs $1 \mathrm{~A}$ to $\mathrm{C}$ : $(\mathrm{A})$ Radiograph depicting fracture reduction and fixation with miniplates, $(\mathrm{B})$ radiograph depicting infection, nonunion and sequestrum formation at the fixation site (plates removed) and $(C)$ radiograph depicting sequestrectomy followed by fracture reduction and fixation with reconstruction plate 

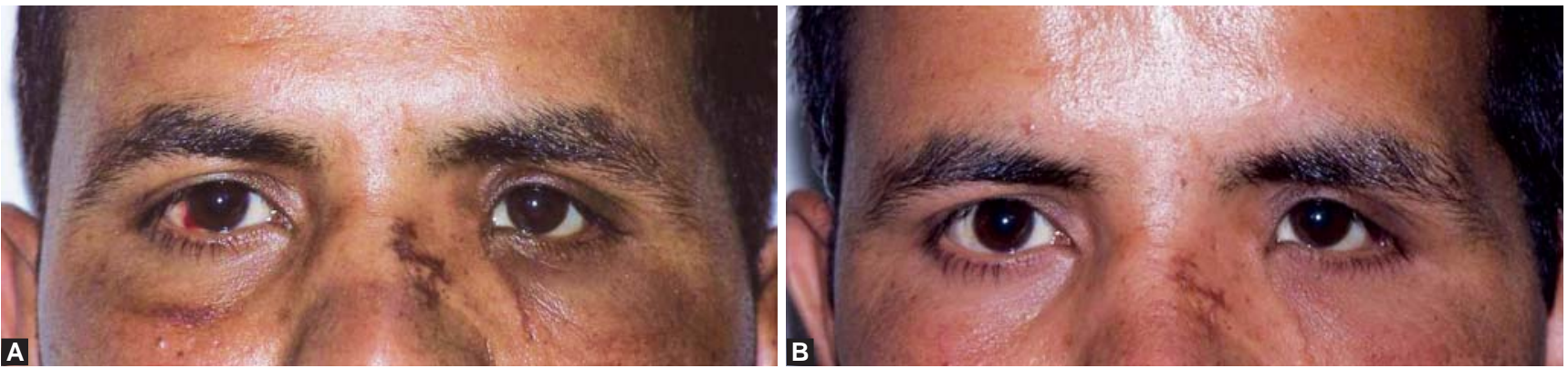

Figs 2A and B: Pre- and postoperative picture of patient who underwent surgery for correction of telecanthus and mongloid slant through bicoronal approach. Pre- and postoperative pictures look same with no improvement despite extensive surgical procedure
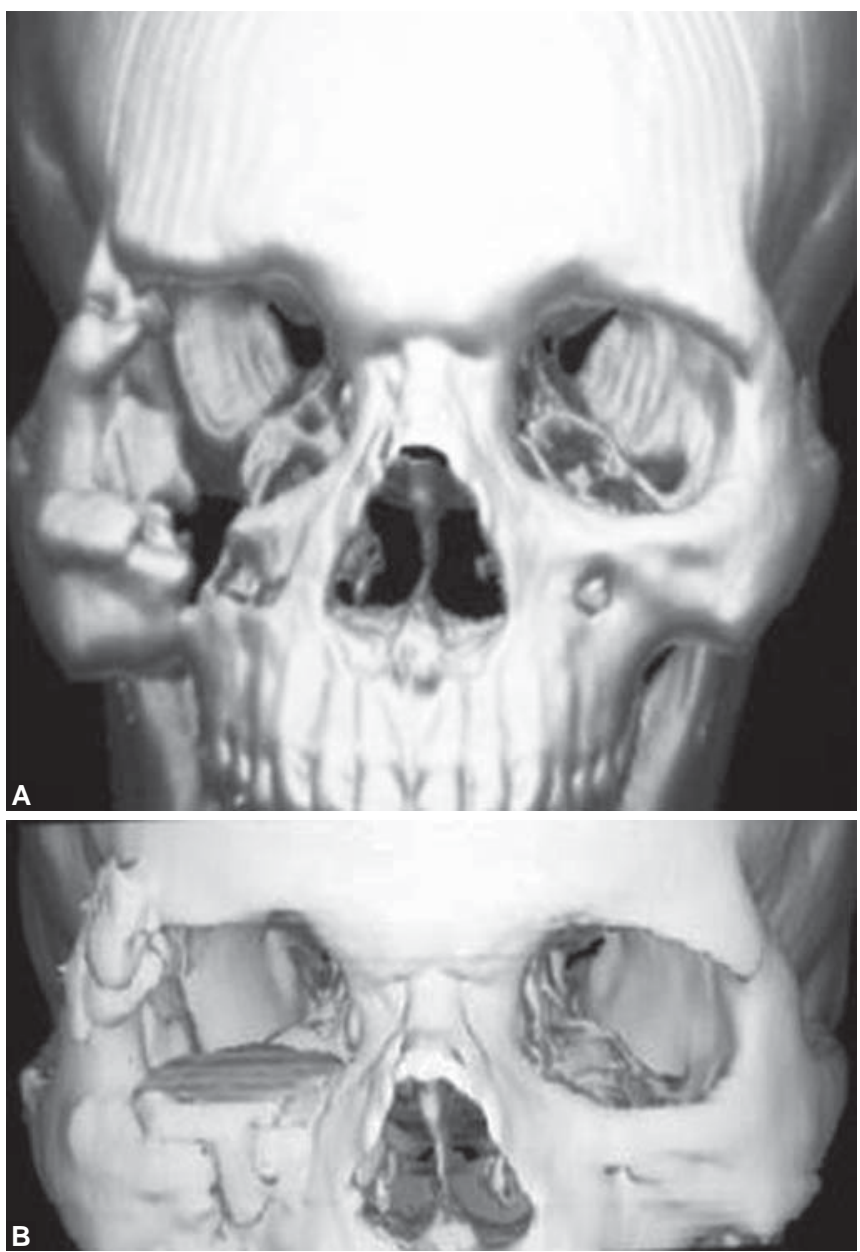

Figs 3A and B: 3D CT depicting orbital floor fracture after malunited zygomatic complex fracture reconstructed by zygomatic osteotomy and calvarial bone graft

of telecanthus to preinjury status is challenging and even after repositioning bone and medial canthus ligament to correct anatomical position results may be compromised (Figs 2A and B). Orbital deformity due to misalignment or nontreatment of orbital wall fractures is a very frequently encountered complication. The management of post-traumatic enophthalmos and aberrant eye position is based on esthetic access incisions, followed by full exploration of orbit and reconstruction of orbital walls (Figs $3 \mathrm{~A}$ and $\mathrm{B}$ ). The aim is to establish correct three-dimensional facial skeleton. ${ }^{5}$ Facial deformities can be managed by osteotomising bone and placing it in correct position, camouflage procedures, augmentation with bone grafts and alloplastic materials, etc. (Figs 4A to F). Replacement of bony and soft tissues and repositioning of malposed tissues to its correct anatomical place can correct the deformities to great extent. Use of computerized planning, stereolithography, intraoperative imaging, intraoperative navigation and customized alloplastic implants can help in achieving predictable results in difficult cases.

\section{OPHTHALMIC COMPLICATIONS}

Inspection of eyes and orbit can be quite challenging in patients of maxillofacial trauma. Preoperative assessment of ophthalmic injury is very crucial with respect to management and avoiding complications. An early ophthalmological consultation should be sought whenever injury to globe is suspected. Early complications related to ophthalmic injuries can be traumatic optic neuropathy, retrobulbar hematoma, globe rupture, vision loss, diplopia, muscle entrapment, enophthalmos, corneal abrasion, superior orbital fissure syndrome, orbital emphysema, blindness, sympathetic ophthalmia. Late complications can be persistent diplopia, enophthalmos, exophthalmos, lower lid malposition, exposure keratitis, blindness, sympathetic ophthalmia, etc. Lower-lid malpositions include scleral show, lower-lid retraction, ectropion and entropion. Injury to contents of superior orbital fissure from direct or indirect trauma may lead to superior orbital fissure syndrome ${ }^{6}$ (Fig. 5). Typically, when blindness is present with superior orbital syndrome, it may lead to orbital apex syndrome.

Recent surveys of craniofacial trauma data suggest an incidence of traumatic optic nerve injury in 2 to $5 \%{ }^{7}$ Traumatic optic nerve injuries can be because of direct or indirect injury to nerve. Penetrating objects into the orbit causing direct damage to the optic nerve have the worse prognosis with respect to indirect injury. Some other causes for indirect iatrogenic injury can be heat transmission during electric cauterization of the posterior ethmoidal artery during treatment of epistaxis and mechanical or heat transmission 

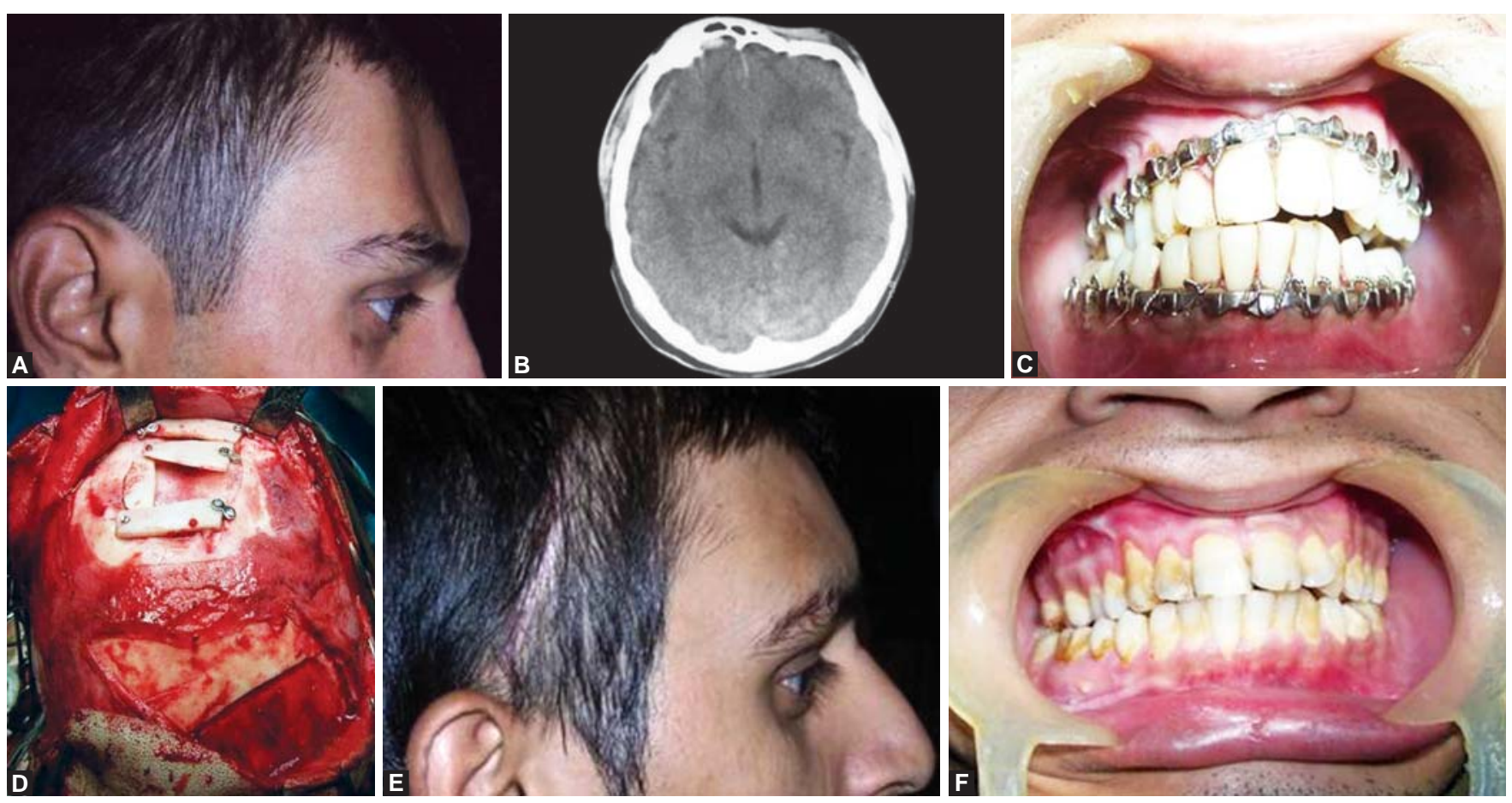

Figs 4A to F: Patient had post-traumatic frontal bone depression and malocclusion due to no surgical intervention initially at the time of trauma. Malocclusion was corrected by Le Forte I osteotomy and frontal bone depression was corrected by calvarial bone graft augmentation

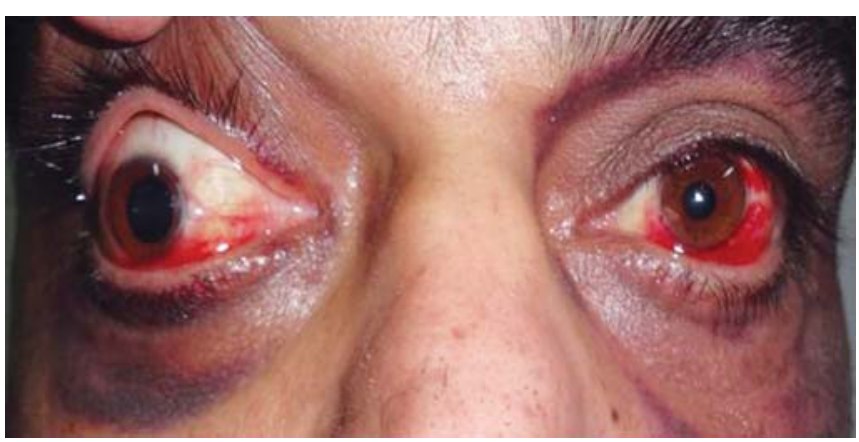

Fig. 5: Traumatic superior orbital fissure syndrome of right eye showing dilated fixed pupil and ophthalmoplegia

injuries during transnasal endoscopic surgery. Patients with direct disruption of the optic nerve will have minimal, if any, recovery. Indirect or secondary injury can be treated and prevented. Treatment includes observation, medical treatment with steroids and optic canal decompression or nerve sheath fenestration. Prompt recognition and early management of vision threatening injuries is crucial for preventing vision loss.

\section{INFECTIONS}

Infection is one of the most commonly encountered complications in relation to trauma. Postoperative infection can be devastating and it can undo all the results achieved during surgery (Figs 6A and B). The causes of postoperative infections are multifactorial. Causes, such as instability, failed hardware, teeth in the line of fracture, ${ }^{8}$ medically compromised patients, delay of treatment and noncompliant patients have been described. Though it may seem easy to be managed, it can have long term systemic and local effects. Following basic principles of surgery and maintaining sterility all through the way while treating the patient, even while changing the dressings, can be of eminent value in preventing it. Early recognition with debridement and adequate antibiotic coverage is essential in managing it. ${ }^{9}$

\section{SOFT AND HARD TISSUE DAMAGE AND LOSS}

Scars are inevitable result of soft tissue trauma or surgical intervention. These can definitely be hidden increases or minimized by using natural openings. Appropriate and early management of scars has a major role to play not only in the functional aspect but has a great deal of psychological impact.

Soft tissue complications, including facial fat atrophy, nerve damage, shortening of the upper lip length, and a widened alar base, can be anticipated as postoperative soft tissue complications.

Parotid fistula can be due to trauma or iatrogenic causes. Amongst various risks and complications associated with transcutaneous surgical approach to mandibular condyle, parotid fistula sialocele is one of them. Surgeon should make sure that at the end of the surgery closure is done in layers and a pressure dressing is placed.

Loss of teeth and alveolar bone are quite common complications secondary to trauma. Dental implants have revolutionized the rehabilitation of dental tissue. Long-term stability of dental implants has been proven and therefore 

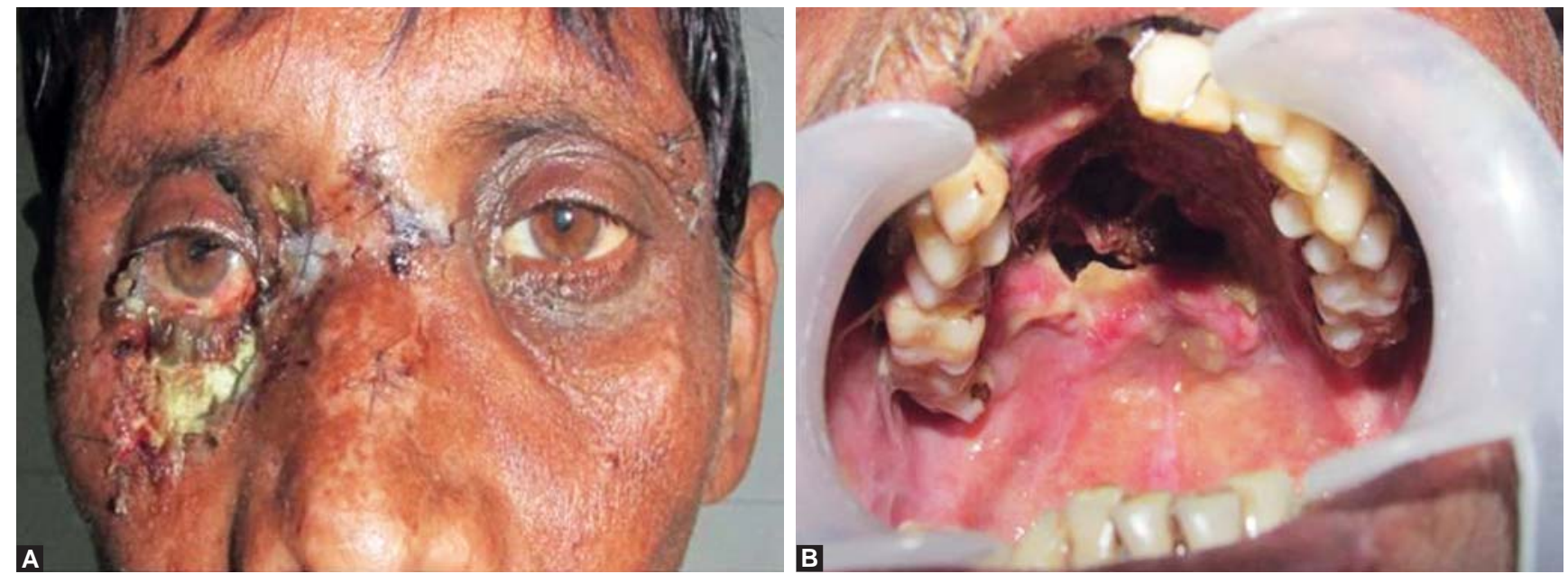

Figs 6 A and B: Patient underwent open reduction and fixation for panfacial fracture. On third postoperative day, infection led to dehiscence of wounds and cause of poor outcome
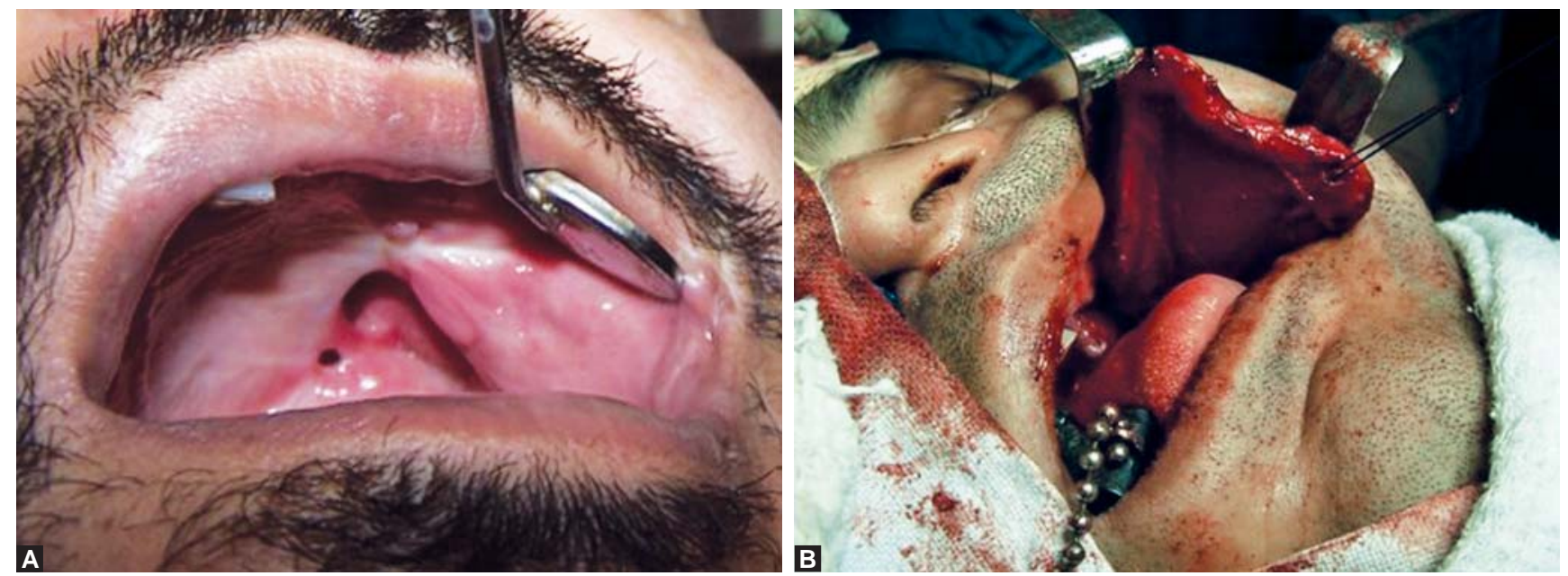

Figs 7A and B: Patient had oronasal communication following loss of maxillary segment due to trauma. Defect was successfully closed with temporalis muscle flap

they are the best treatment option for young patients with missing teeth. Distraction osteogenesis of alveolar bone can be done where there is significant loss of basal or supporting jaw bone.

Missing hard tissue can be replaced with free or vascularized bone grafts and soft tissue cover can be provided with regional or distant flaps (Figs 7A and B). Minor residual deformity can be expected with these modalities but there is significant restoration of form and function. ${ }^{10}$

\section{GROWTH RESTRICTION}

Growth restriction is another complication after trauma in growing children. Unilateral or bilateral condylar fracture, if misdiagnosed or inadequately treated can lead to an open bite and decreased mouth opening eventually leading to a retrognathic appearance. ${ }^{11}$ Temporomandibular joint ankylosis may also develop, resulting in severe growth restriction in children ${ }^{10}$ (Figs 8A and B). Early treatment is required for its release to prevent growth problems.

\section{NERVE INJURY}

The incidence of traumatic cranial nerve injury is approximately 5 to $23 \% .{ }^{12}$ Four factors are known to affect the rate and degree of peripheral sensory nerve recovery following injury which are age, state of general health, location of the injury and type of injury. Facial nerve and trigeminal nerve branches are the most commonly involved nerves in facial trauma.

\section{Olfactory Nerve}

The severity of dysfunction is related to the severity of head injury. The damage to frontotemporal cortical structures or shearing of the olfactory nerve filaments can lead to dysosmia or anosmia. The cribriform plate plays a role in passage of olfactory nerves and trauma to the plate can cause disruption of the nerve and can cause partial or total anosmia. The olfactory pathway continuously rebuilds itself throughout life. More invasive treatment, including removal 

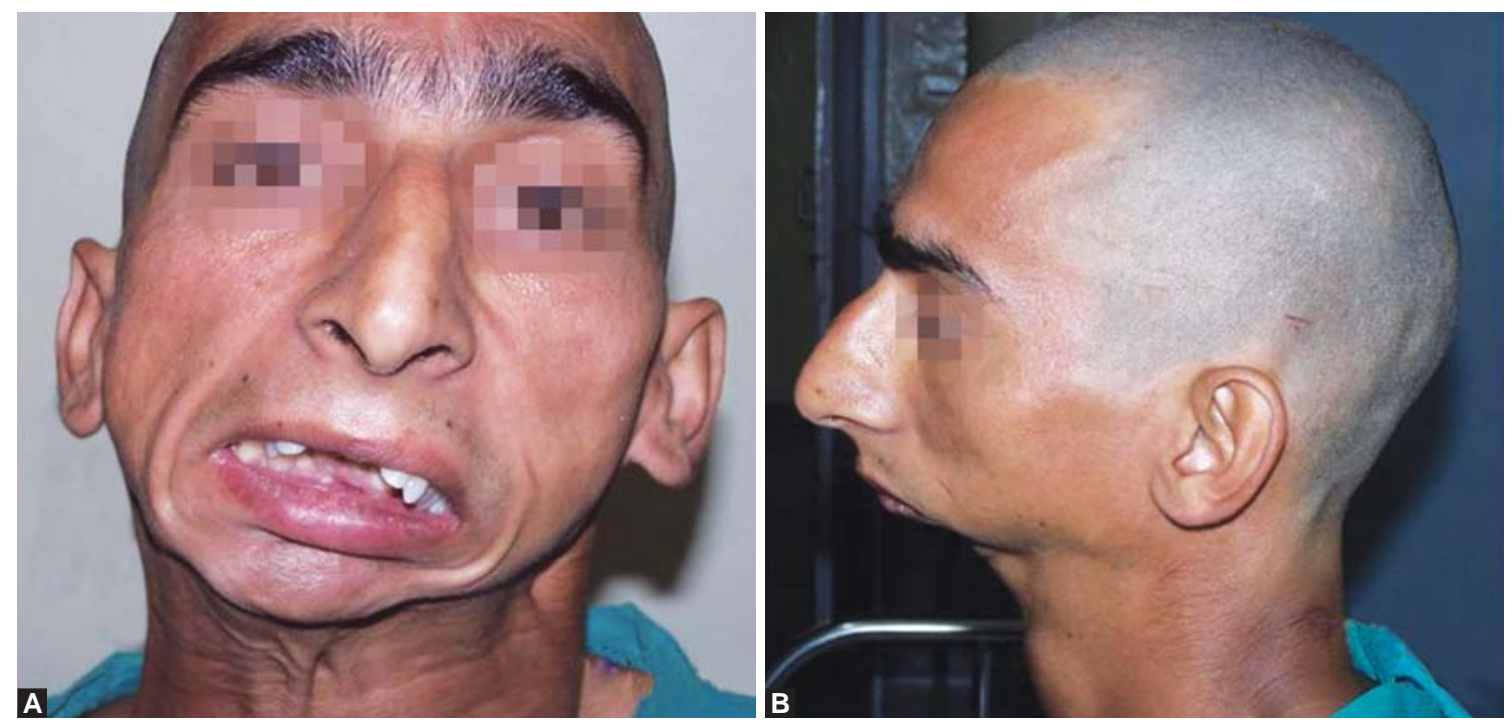

Figs $8 \mathrm{~A}$ and $\mathrm{B}$ : Childhood trauma to the mandibular condyle led to severe facial growth problem

of the olfactory bulbs and tracts, is reserved for patients with severe or permanent smell alteration. ${ }^{13}$

\section{Trigeminal Nerve}

Injury to peripheral branches of the trigeminal nerve can arise from a wide variety of oral and maxillofacial surgical procedures or injuries. It can well be a direct consequence of maxillofacial trauma or secondary to surgical interventions for repair of facial trauma. Trigeminal nerve injuries not only causes significant neurosensory deficits and facial pain, but can cause significant comorbidities due to changes in eating habits from muscular denervation of masticator muscles or altered sensation of the oral mucosa. It is advisable to decompress the nerve with open reduction as closed reductions continue to compress the nerve because of reactive osseous proliferation. The terminal branches affected by trauma are the supraorbital and supratrochlear nerves in the upper face, infraorbital nerve in the midface and inferior alveolar nerve in the lower face. Most fractures of the zygomatic complex will cause some damage to the infraorbital nerve, which may lead to temporary or permanent altered sensation on the distribution of this nerve. ${ }^{12}$ Injuries caused by direct blunt trauma in an undisplaced fracture offer a much better prognosis and can be treated conservatively. Injuries caused by sharp trauma or entrapment by bony fragments have a more reserved prognosis and should be treated surgically, especially in the presence of a displaced fracture. However, the surgical treatment itself carries the risk of causing further injury to the nerve.

\section{Facial Nerve}

Facial nerve injury usually results from blunt or penetrating trauma to the petrous portion of the temporal bone. Facial nerve weakness can be classified as partial or complete; manifesting immediately or in a delayed fashion. Reasons behind late presentation can be pressure effect from hemorrhage, edema, or granulation tissue. Evaluation can be challenging, especially in the acute trauma setting. Battle's sign, cerebral spinal fluid otorrhea, air or fluid in the mastoid air cells or fracture of the temporal bone should alert the clinician to further pursue any facial nerve injury. High resolution CT scan of temporal bone may be helpful in assessing the site of injury and decompression of the facial nerve may aid in recovery. Patients with paresis can be managed with observation. Medical therapy in the form of steroids may be helpful in certain cases.

In conclusion proper diagnosis, appropriate treatment plan, proper execution of plan, postoperative assessment and reassessment go a long way in preventing complications. If any complication is recognized, an early suitable intervention should be sought. Team approach is one of the most important keys to minimize post-traumatic maxillofacial complications. Expertise of various specialists in management of complicated cases should be sought. Team members must show respect and confidence in capabilities of other specialists and each specialist must acknowledge its own limitations and take help from other specialists for best outcome.

\section{REFERENCES}

1. Ellis E. Complications of rigid internal fixation for mandibular fractures. J Craniomaxillofac Trauma 1996;2(2):32-39.

2. Kawamoto HK. Correction of established traumatic deformities of the facial skeleton using craniofacial principles. In: Schultz RC, editor. Facial Injuries. Chicago, IL, Yearbook, 1988. p. 601-630.

3. Zachariades N, Papademetriou I, Rallis G. Complications associated with rigid internal fixation of facial bone fractures. J Oral Maxillofac Surg 1993;5:275-278. 
4. Bell RB, Kindsfater CS. The use of biodegradable plates and screws to stabilize facial fractures. J Oral Maxillofac Surg 2006; 64(1):31-39.

5. Koo L, Hatton M, Rubin P. When is enophthalmos significant? Ophthal Plast Reconstr Surg 2006;22(4):274-277.

6. Rai S, Rattan V. Traumatic superior orbital fissure syndrome: review of literature and report of three cases. Natl J Maxillofac Surg 2012;3:222-225.

7. Urolagin SB, Kotrashetti SM, Kale TP, Balihallimath LJ. Traumatic optic neuropathy after maxillofacial trauma: a review of 8 cases. J Oral Maxillofac Surg 2012 May;70(5):11231130.

8. Rai S, Pradhan R. Tooth in the line of fracture: its prognosis and effects on healing. Indian J Dent Res 2011;22:495-496.

9. Benson PD, Marshall MK, Engelstad ME, et al. The use of immediate bone grafting in reconstruction of clinically infected mandibular fractures: bone grafts in the presence of pus. J Oral Maxillofac Surg 2006;64(1):122-126.

10. Rattan V. A simple technique for use of buccal pad of fat in temporomandibular joint reconstruction. J Oral Maxillofac Surg 2006 Sep;64(9):1447-1451.

11. Belli E, Matteini C, Incisivo V. Orthodontic-surgical treatment after post-traumatic bilateral condylectomy of the mandible in an adult patient. J Craniofac Surg 2003;14:55.

12. Schultze-Mosgau S, Erbe M, Rudolph D, Ott R, Neukam FW. Prospective study on post-traumatic and postoperative sensory disturbances of the inferior alveolar nerve and infraorbital nerve in mandibular and midfacial fractures. J Craniomaxillofac Surg 1999 Apr; 27(2):86-93.

13. Haxel BR, Grant L, Mackay-Sim A. Olfactory dysfunction after head injury. J Head Trauma Rehabil 2008 Nov-Dec;23(6): 407-413. 